\title{
Cardiovascular magnetic resonance imaging of aorto-iliac and ilio-femoral vascular calcifications using proton density-weighted in-phase stack of stars
}

Ali Serhal ${ }^{1,2}$, Ioannis Koktzoglou ${ }^{2,3}$, Pascale Aouad ${ }^{1}$, James C. Carr ${ }^{1}$, Shivraman Giri ${ }^{4}$, Omar Morcos ${ }^{5}$ and Robert R. Edelman ${ }^{1,2^{*}}$ (D)

\begin{abstract}
Background: Comparing cardiovascular magnetic resonance (CMR) angiography with computed tomography angiography (CTA), a major deficiency has been its inability to reliably image peripheral vascular calcifications that may impact the choice of interventional strategy and influence patient prognosis. Recently, MRI using a proton density-weighted, in-phase stack of stars (PDIP-SOS) technique has proved capable of detecting these calcifications. The goal of the present study was two-fold: (1) to determine whether magnetic field strength impacts the apparent size and conspicuity of ilio-femoral arterial calcifications; and (2) to determine whether the technique can be accurately applied to image aorto-iliac arterial calcifications.

Main body: Two patient cohorts were studied. For the first cohort, ilio-femoral arterial calcifications were imaged at 1.5 Tesla in 20 patients and at 3 Tesla in 12 patients. For the second cohort, aorto-iliac arterial calcifications were imaged in 10 patients at 3 Tesla and one patient at 1.5 Tesla. Qualitative image analysis as well as quantitative analysis using a semi-automated technique were performed using CTA as the reference standard.

Qualitatively, most PDIP-SOS CMR images showed good-to-excellent confidence to detect vascular calcifications, with good-to-excellent inter-reader agreement ( $\mathrm{k}=0.67$ for ilio-femoral region, $P<0.001 ; \mathrm{K}=0.80$ for aorto-iliac region, $P<0.01)$. There was an overall excellent correlation $(r=0.98, P<0.001)$ and agreement (intraclass correlation coefficient $=0.97, P<0.001$ ) between PDIP-SOS CMR and CTA measures of calcification volume in both regions, with no overt difference in performance at 1.5 Tesla vs. 3 Tesla for ilio-femoral calcifications. CMR lesion volumes were slightly lower than those measured for CTA.
\end{abstract}

Conclusion: Using PDIP-SOS CMR, aorto-iliac and ilio-femoral calcifications could be simultaneously evaluated at 3 Tesla in less than six minutes with excellent correlation and agreement to CTA. Our results suggest that PDIP-SOS CMR provides a reliable alternative to $C T$ for pre-interventional evaluation of peripheral vascular calcium burden.

Keywords: Vascular calcification, Stack of stars, Magnetic resonance imaging, Quiescent-interval slice-selective, CT angiography, Peripheral arterial disease

\footnotetext{
* Correspondence: redelman999@gmail.com

${ }^{1}$ Radiology, Northwestern Memorial Hospital, Chicago, IL, USA

${ }^{2}$ Radiology, Northshore University HealthSystem, Walgreen Building, G534,

2650 Ridge Avenue, Evanston, IL 60201, USA

Full list of author information is available at the end of the article
}

(c) The Author(s). 2018 Open Access This article is distributed under the terms of the Creative Commons Attribution 4.0 International License (http://creativecommons.org/licenses/by/4.0/), which permits unrestricted use, distribution, and reproduction in any medium, provided you give appropriate credit to the original author(s) and the source, provide a link to the Creative Commons license, and indicate if changes were made. The Creative Commons Public Domain Dedication waiver (http://creativecommons.org/publicdomain/zero/1.0/) applies to the data made available in this article, unless otherwise stated. 


\section{Background}

Both computed tomography angiography (CTA) and cardiovascular magnetic resonance (CMR) angiography are accurate tests for the cross-sectional assessment of peripheral arterial disease (PAD) [1, 2]. Compared with CMR angiography, CTA offers higher speed, lower cost, and fewer drawbacks overall. Nonetheless, peripheral CMR angiography remains in widespread use, in part because it avoids exposure to ionizing radiation and, given that patients with PAD often suffer from poor renal function [3], offers the option of imaging without an exogenous contrast agent [4].

In diabetic patients, CMR angiography is advantageous compared with CTA because of the high prevalence of peripheral vascular calcifications [5]. With CTA, these calcifications confound the evaluation of small caliber vessels due to blooming artifact that obscures the vessel lumen [6]. Since vascular calcifications are unapparent and do not cause image artifacts with CMR angiography, it is often preferred to CTA in this patient group. However, important information deriving from the presence of vascular calcifications, including the impact on interventional strategy and patient prognosis, is then lost [7-10]. For instance, dense arterial wall calcifications should be avoided when choosing a percutaneous access site, and their presence is a major determinant of failure for percutaneous endovascular aneurysm repair [10].

The inability to image vascular calcifications remains an important and unaddressed deficiency of CMR angiography. For instance, in the largest clinical trial to evaluate the association of plaque characteristics with functional performance in patients with PAD, investigators were unable to reliably identify vascular calcifications [11]. The only way to recover this information would be to perform an additional CT scan, which is inconvenient and costly.

Vascular calcifications have negligible signal intensity with standard CMR pulse sequences due to low free water concentration and short $\mathrm{T}^{*}$ [12]. While this property makes them difficult to visualize with CMR angiography, a recent study demonstrated the feasibility of using "neutral contrast" 3D gradient-echo techniques to depict peripheral vascular calcifications on minimum intensity projection CMR images [13]. However, a major unknown with using CMR to image vascular calcifications is the potential impact of field strength on apparent lesion volumes. Just as calcifications can show blooming artifact with $\mathrm{CT}$, diamagnetic susceptibility and $\mathrm{T} 2 \%$ effects have the potential to cause field strength-dependent blooming artifact with CMR. Vascular calcifications are strongly diamagnetic - as much as ten times that of the vessel wall which results in field strength-dependent phase shifts [14]. Moreover, the signal-to-noise ratio (SNR), R2*, chemical shift and off-resonance effects all increase with field strength.

Another unknown is whether CMR can reliably image vascular calcifications in the abdomen and pelvis. In these regions, respiratory motion as well as presence of peristalsing, air-containing bowel loops have the potential to cause blurring and off-resonance effects, thereby obscuring vessel calcifications.

To address these concerns, the goals of the present study were two-fold: (1) to determine whether magnetic field strength impacts the apparent size and conspicuity of ilio-femoral arterial calcifications; and (2) to determine whether the technique can be applied to accurately image aorto-iliac arterial calcifications.

\section{Methods}

This prospective study was approved by the Institutional Review Boards of two academic centers and informed consent was obtained. Two cohorts of patients were studied. For the first cohort, 32 patients (52-84 years, 10 female) were consecutively recruited from those in whom CTA performed within the preceding three months demonstrated vascular calcifications in at least one ilio-femoral vessel segment. At one institution, 20 patients were imaged using a 1.5 Tesla MAGNETOM Aera scanner (Siemens Healthineers, Erlangen, German), while at the other institution 12 patients were imaged using a 3.0 Tesla MAGNETOM Skyra Fit scanner (Siemens Healthineers). For the second cohort, 11 patients (57-83 years, 2 female) were recruited from those in whom CTA demonstrated vascular calcifications in the region of the aorto-iliac bifurcation; ten patients in this cohort were imaged at 3 Tesla, while one patient was imaged at 1.5 Tesla.

Nonenhanced quiescent-interval slice-selective (QISS) CMR angiography [15] of the peripheral arteries was used as the scout for positioning the proton density-weighted, in-phase stack-of-stars (PDIP-SOS) spoiled gradient-echo pulse sequence. This sequence provides nearly isotropic images in which muscle, fat, and intravascular signal show intermediate signal intensity, whereas vascular calcifications appear uniformly dark [16]. Unlike a Cartesian 3D acquisition, in which chemical shift artifacts at fat/water interfaces appear as discrete dark lines that can obscure or be confounded with vascular calcifications in minimum intensity projections, these interfaces are less distinct with the in-phase stack-of-stars radial k-space trajectory so that they are invisible in the projection images.

For the first cohort of patients, the PDIP-SOS sequence used a legacy encoding scheme whereby all radial views were collected in rapid sequence as a single shot. For the second cohort of patients, the k-space 
sampling strategy was updated so that all slice partitions, rather than radial views, were collected in rapid sequence.

PDIP-SOS images were acquired in an oblique coronal plane. Body and peripheral phased array coils were used for signal reception. There were some differences in the imaging parameters between the two magnetic field strengths, due primarily to the in-phase echo time at 1.5 Tesla being twice the value at 3 Tesla and the use of a lower sampling bandwidth to improve the SNR, in turn lengthening the repetition time. Consequently, some compromises in spatial resolution were needed at 1.5 Tesla to avoid increasing scan time and to maintain adequate SNR despite the lower field strength. Slice thickness was $1.3-\mathrm{mm}$ at 1.5 Tesla and $1.0-\mathrm{mm}$ at 3 Tesla, with 128 reconstructed slices per 3D slab and in-plane resolution at both field strengths of $1.0-\mathrm{mm} \times$ $1.0-\mathrm{mm}$ prior to interpolation. For the second patient cohort using the updated k-space sampling strategy, PDIP-SOS CMR encompassing both the aorto-iliac and ilio-femoral regions was acquired with 1200 radial views in a scan time of $5 \min 49 \mathrm{~s}$. The PDIP-SOS images were processed into thin (4 to $15-\mathrm{mm}$ ) minimum intensity projections for qualitative display of the vascular calcifications.

The CTA studies were acquired on three different scanners: SOMATOM Force, SOMATOM Definition AS and SOMATOM Definition (Siemens Healthineers, Forchheim, Germany) with standard technique using either iterative reconstruction or filtered back projection according to clinical routine. Typical slice thickness was on the order of $0.6-\mathrm{mm}$ to $1-\mathrm{mm}$. $\mathrm{kVp}$ was selected according to the pre-determined body mass index-based institutional protocol.

\section{Quantitative image analysis}

Quantification of the calcification volumes was done using in-house software executed within ImageJ (version 1.51p, National Institutes of Health, Bethesda, Maryland, USA). For analysis of the ilio-femoral region, on each lower limb extremity, two segments of the femoral artery were analyzed: the proximal segment which covered $5 \mathrm{~cm}$ of the common femoral artery immediately above the femoral bifurcation, and the distal segment which covered $5 \mathrm{~cm}$ of the superficial femoral artery immediately below the bifurcation. For analysis of the aorto-iliac region, five segments of the aorta and iliac vessels were analyzed: the most distal $5 \mathrm{~cm}$ segment of the aorta, the left and right common iliac arteries, and the left and right external iliac arteries. The segmentation algorithm volumetrically segmented the calcifications using thresholds based on Hounsfield units for CTA images and on signal intensity for CMR images. Using this algorithm, we have previously found that a threshold of 560
Hounsfield units was optimal for distinguishing calcifications on CTA from contrast-enhanced lumen. For CMR images of the ilio-femoral region, voxels having a signal intensity more than three standard deviations below the mean were classified as calcifications. A CMR threshold of two (instead of three) standard deviations was used in the aorto-iliac region due to slightly increased background signal variability in this region from bowel and respiratory motion. Bicubic interpolation was used to scale all CMR and CTA data to $0.5 \mathrm{~mm}$ isotropic resolution before measurement of calcification volume.

\section{Qualitative image analysis}

Two cardiovascular radiology fellows (AS, PA), blinded to clinical information, qualitatively reviewed source images and multi-planar reformats for both CTA and CMR. CTA images were taken as a reference and the PDIP-SOS CMR images were analyzed for degree of confidence to detect calcification, matching size, shape and location of calcification with the CTA images. Images were scored using a 5-point Likert scale as: 1- very poor, 2- poor, 3- fair, 4- good, 5- excellent.

\section{Data and statistical analysis}

Correlation between volume of vascular calcification shown by PDIP-SOS and CTA was assessed by linear regression and Pearson's correlation analysis (with correlation coefficient, $r$ ). Agreement of vascular calcification volume was assessed by intraclass correlation coefficient (ICC). Sub-analyses of data in the ilio-femoral region were performed by stratifying according to magnetic field strength (1.5 Tesla and 3 Tesla), and severity of calcification (none or mild, moderate, severe) based on calcification volume measures on CTA. Group 1 included patients with no or mild vascular calcification (volume 0-99 $\mathrm{mm}^{3}$ ), group 2 included patients with moderate vascular calcification (volume $100-299 \mathrm{~mm}^{3}$ ), and group 3 included patients with severe vascular calcification (volume $\geq 300 \mathrm{~mm}^{3}$ ). Weighted Cohen's kappa (к) coefficient was used to assess inter-rater agreement of qualitative scores. Pearson correlation (r), ICC, and $\mathrm{k}$ coefficients were interpreted as follows: $<0.40-$ poor, 0.40-0.59 - fair, 0.60-0.74 - good, $\geq 0.75$ - excellent [17]. Bland-Altman analysis was used to compute mean bias (MR volume minus CTA volume) and 95\% limits of agreement. Statistical analyses were performed in $\mathrm{R}$ software (version 3.4.2, R Foundation for Statistical Computing, Vienna, Austria).

\section{Results}

\section{Cohort 1 (llio-femoral vessels)}

Of 128 ilio-femoral vessel segments available for analysis, 6 were excluded (4 due to magnetic susceptibility artifact from hip prosthesis or stent and 2 due to artifact 
relating to recent bypass graft), leaving a total of 122 vessel segments that were analyzed.

Qualitatively, PDIP-SOS CMR images showed good-to-excellent confidence to detect ilio-femoral vascular calcifications with a mean score of 4.1 (range 3-5) for the first reader and 4.3 (range 3-5) for the second reader. Inter-rater agreement was good $(\kappa=0.67,95 \%$ confidence interval (CI): $0.54-0.80, P<0.001)$. The size, shape and location of the calcifications by PDIP-SOS CMR matched CTA findings in all patients regardless of lesion volume (Fig. 1).

Figure 2 shows scatter plots of calcification volume in the ilio-femoral region stratified by magnetic field strength and severity of calcification. There was an overall excellent correlation $(r=0.98,95 \% \mathrm{CI}$ : 0.97-0.99, $P<0.001)$ and agreement $(\mathrm{ICC}=0.97,95 \% \mathrm{CI}: 0.96-$ 0.98, $P<0.001$ ) between PDIP-SOS CMR and CTA measures of calcification volume (Fig. 2a). The average volume was $124 \mathrm{~mm}^{3}$ (range 0-953 $\mathrm{mm}^{3}$ ) by CTA and $109 \mathrm{~mm}^{3}$ (range $0-914 \mathrm{~mm}^{3}$ ) by CMR. When comparing the results of CMR exams done on 1.5 Tesla and 3 Tesla, the correlation coefficients were $r=0.98$ (95\% CI: $0.97-$
0.99, $P<0.001)$ and 0.99 (95\% CI: 0.98-0.99, $P<0.001)$, while ICC values were 0.96 (95\% CI: 0.94-0.97, $P<0.001)$ and 0.99 (95\% CI: $0.98-0.99, \quad P<0.001)$, respectively (Figs. 2b and c). Bland-Altman mean biases and 95\% limits of agreement (CMR minus CTA calcification volume) at both magnetic field strengths, 1.5 Tesla, and 3 Tesla were $-15.7[-95.2,63.8] \mathrm{mm}^{3},-20.3[-107.9$, $67.3] \mathrm{mm}^{3}$, and $-7.0[-65.4,51.4] \mathrm{mm}^{3}$, respectively.

The Pearson correlation coefficients of calcification volume for patients with no-to-mild, moderate and severe vascular calcifications were $r=0.83$ (95\% CI: 0.74-0.89), 0.90 (95\% CI: 0.80-0.95), and 0.88 (95\% CI: $0.69-0.96)(P<0.001$ for all), respectively (Figs. $2 d-f)$. Corresponding ICC values for agreement in these three strata remained in the excellent range and were 0.82 (95\% CI: 0.73-0.88), 0.77 (95\% CI: 0.58-0.89), and 0.84 (95\% CI: $0.63-0.94)(P<0.001$ for all), respectively. Bland-Altman mean biases [95\% limits of agreement] for patients with no-to-mild, moderate, and severe vascular calcifications were $-1.7[-30.0,26.6]$ $\mathrm{mm}^{3},-30.3[-80.5,19.9] \mathrm{mm}^{3}$, and $-53.4[-223.1$, 116.3] $\mathrm{mm}^{3}$, respectively.

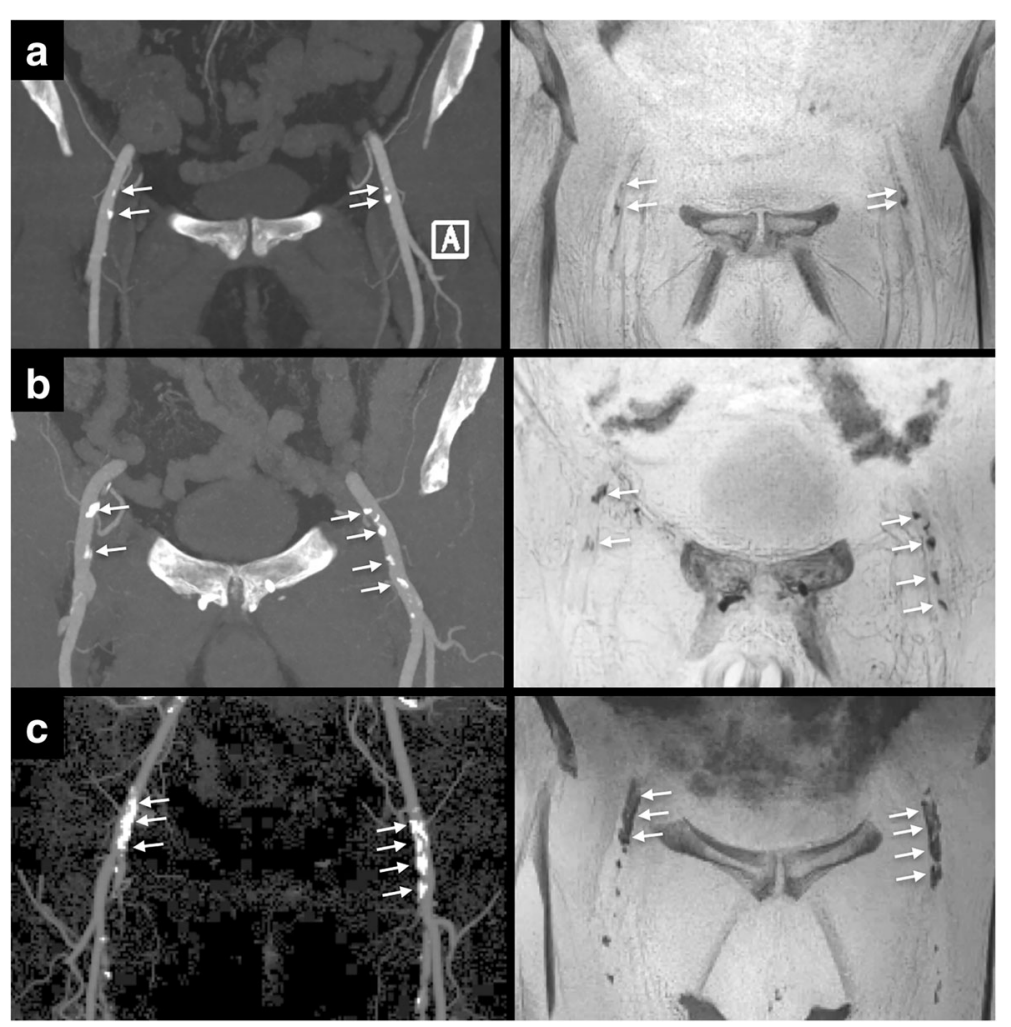

Fig. 1 Examples of PDIP-SOS CMR (right) in comparison to CTA (left) for: (a) 66-year-old female imaged at 1.5 Tesla showing mild vascular calcifications (Group 1), (b) 80-year-old male imaged at 1.5 Tesla showing moderate vascular calcifications (Group 2), and (c) 68-year-old female imaged at 3 Tesla showing severe vascular calcifications (Group 3). Vascular calcifications (arrows) appear dark with PDIP-CMR (presented as thin minimum intensity projections), and bright with CTA (presented as thin maximum intensity projections). There is excellent correlation between CMR and CTA irrespective of lesion severity 


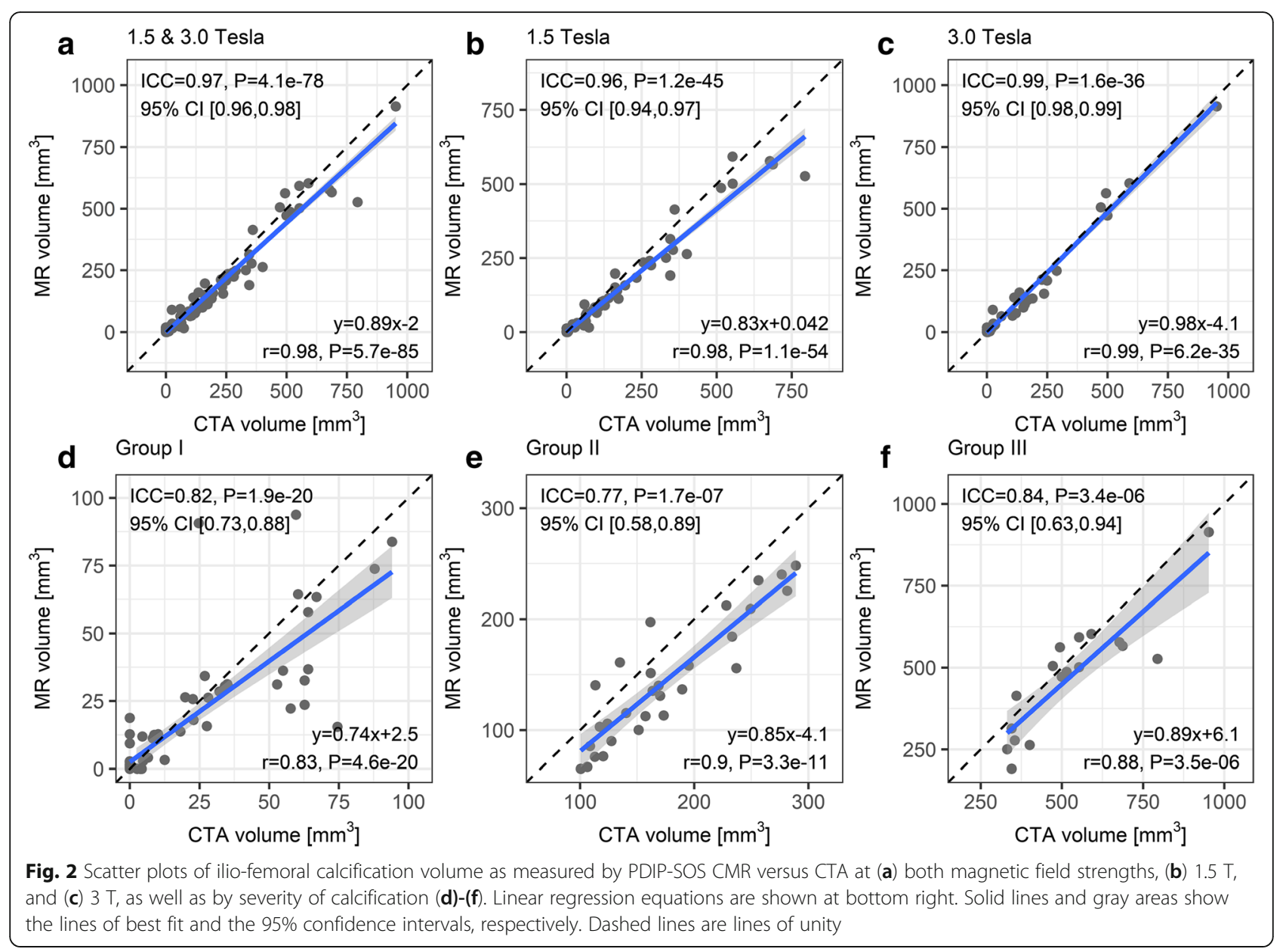

\section{Cohort 2 (aorto-iliac vessels)}

Initial studies demonstrated that the order in which the slice partitions and radial views were collected, while largely irrelevant for the ilio-femoral vessels, had a profound effect on image quality for the aorto-iliac vessels. Acquisitions using a legacy k-space encoding strategy in which the radial views were collected in rapid sequence as a single shot typically showed extensive artifacts relating to air-containing bowel loops which often obscured the distal aorta and pelvic arteries. In contrast, these artifacts were largely absent when the slice partitions were collected in rapid sequence as a single shot allowing for unambiguous identification of vascular calcifications (Fig. 3).

A total of 55 aorto-iliac vessel segments were analyzed, and none were excluded. Qualitatively, most PDIP-SOS CMR images showed good-to-excellent confidence to detect aorto-iliac vascular calcifications with a mean score of 4.1 (range 3-5) for the first reader and 4.0 (range 3-5) for the second reader. Inter-rater agreement was excellent $(\mathrm{K}=0.80,95 \%$ confidence interval $(\mathrm{CI})$ : $0.57-1.00, P<0.01)$.
Figure 4 shows a scatter plot of calcification volume in the aorto-iliac region. There was an overall excellent correlation $(r=0.98,95 \%$ CI: $0.97-0.99, P<0.001)$ and agreement $(\mathrm{ICC}=0.97,95 \%$ CI: $0.96-0.98, P<0.001$ ) between PDIP-SOS CMR and CTA measures of calcification volume. The average volume was $450 \mathrm{~mm}^{3}$ (range $0-2750 \mathrm{~mm}^{3}$ ) by CTA and $391 \mathrm{~mm}^{3}$ (range 0$2854 \mathrm{~mm}^{3}$ ) by CMR. The Bland-Altman (CMR minus CTA calcification volume) mean bias was $-58.5 \mathrm{~mm}^{3}$, while the $95 \%$ limits of agreement were $[-261.8$, 144.7] $\mathrm{mm}^{3}$.

\section{Discussion}

Prior work has shown that CMR using a PDIP-SOS pulse sequence is accurate for ilio-femoral vascular calcifications [16]. The present work differs in three significant ways from this prior work. First, the present study was sufficiently powered to examine the impact of magnetic field strength (1.5 Tesla vs. 3 Tesla) on sequence performance and apparent lesion size for ilio-femoral calcifications. Second, we used a new motion-resistant $\mathrm{k}$-space trajectory to additionally enable evaluation of 


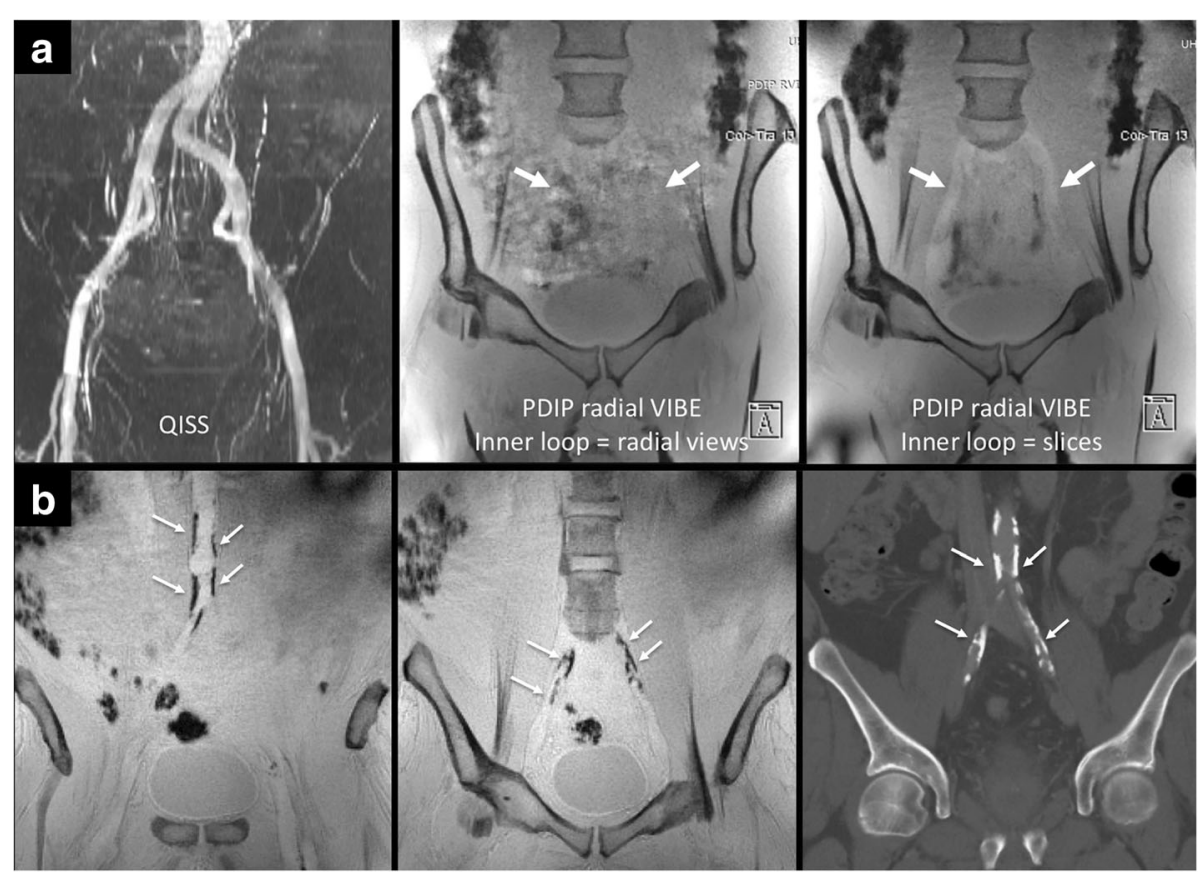

Fig. 3 Evaluation of the aorto-iliac vessels using PDIP-SOS CMR. (a) Healthy volunteer. Left: QISS nonenhanced CMR angiogram used for positioning the stack of stars imaging slab. Middle: PDIP-SOS CMR using the legacy k-space encoding approach of acquiring all radial views in rapid sequence. There are severe ghost artifacts arising from centrally-located bowel loops that obscure the iliac vessels (arrows). Right: Identical PDIP-SOS acquisition except that all slice partitions (instead of radial views) were acquired in rapid sequence. The bowel-related artifacts are eliminated, so that the iliac vessels are well shown (arrows). Note that both the aorto-iliac and ilio-femoral regions are encompassed in the field of view, allowing simultaneous assessment of both regions. (b) Patient with aorto-iliac calcifications. Left, middle: 4-mm thick minimum intensity projections from PDIP-SOS CMR clearly depict vascular calcifications involving the distal aorta and proximal iliac vessels. Right: Thin-slab maximum intensity coronal projection from the CT shows excellent correspondence with the PDIP-SOS CMR

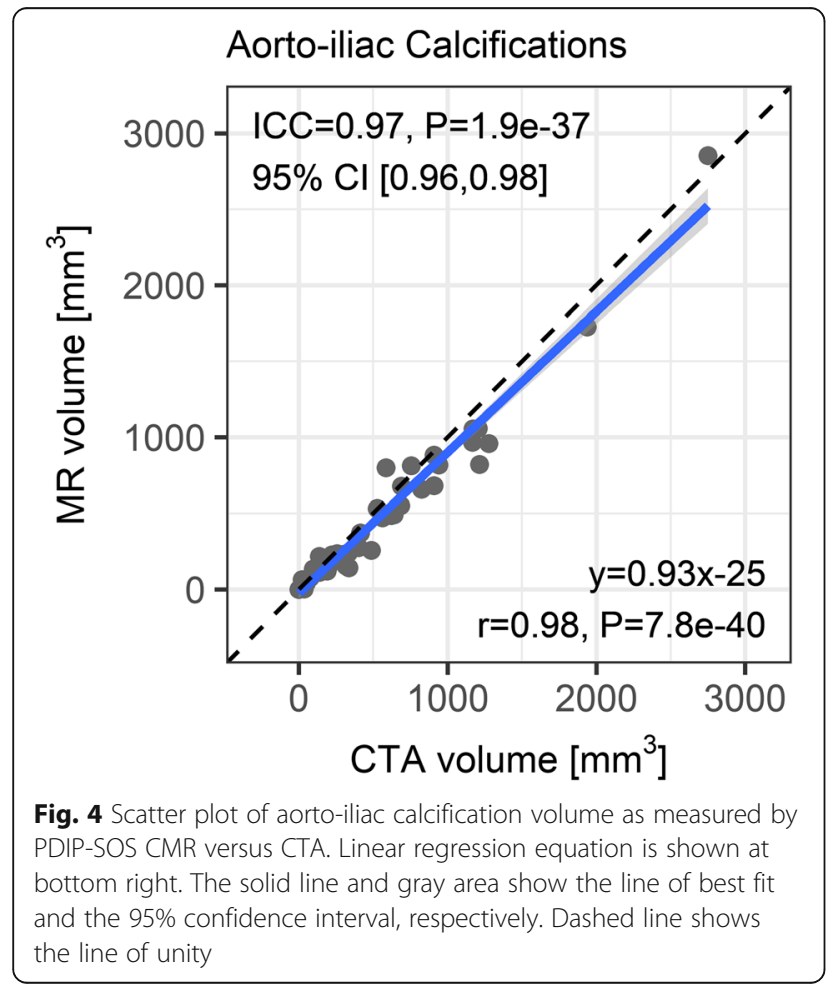

aorto-iliac calcifications, which was not possible with earlier versions of the technique due to excessive respiratory and bowel motion artifacts. Third, a new cohort of patients with PAD was used for the present study with no overlap to the prior study.

Our results indicate that CMR using a PDIP-SOS pulse sequence depicts ilio-femoral and aorto-iliac calcifications with excellent correlation $(r \geq 0.98)$ and agreement (ICC $\geq 0.97)$ to CTA over a wide range of lesion volumes. The correlation and agreement to CTA for ilio-femoral calcifications were similar at 1.5 Tesla and 3 Tesla, suggesting that lesion volumes as measured by PDIP-SOS CMR are not substantially dependent on magnetic field strength. Regardless of field strength, in no case did CMR miss large calcifications that might adversely impact the outcome of a percutaneous procedure, nor falsely suggest significant calcifications where none existed in the CTA.

Given that CMR is generally considered to be insensitive to vascular calcifications, it is notable that the PDIP-SOS imaging technique could detect calcifications over a wide range of lesion volumes, including small vascular calcifications that spanned only a few pixels. However, the use of a very small voxel (e.g. $\sim 1 \mathrm{~mm}^{3}$ in 
the current study) and adequate SNR are essential for confidently detecting small lesions.

The stack of stars k-space trajectory has the benefit of being less sensitive to artifacts from respiratory motion than a Cartesian 3D acquisition, due to extensive oversampling of the center of k-space [18]. Nonetheless, we found that our legacy k-space encoding approach for PDIP-SOS, in which all radial views were acquired in rapid sequence as a single shot, often showed severe image artifacts in the pelvic and abdominal regions due to respiratory and peristaltic motion of bowel loops. These bowel-related artifacts often obscured nearby vascular calcifications. In order to overcome these artifacts, we modified the k-space sampling strategy to acquire all slice partitions in rapid sequence rather than radial views. A similar sampling strategy has previously been reported to reduce respiratory motion artifact in the upper abdomen [19], and we found it to be highly effective at suppressing artifacts from bowel motion in the lower abdomen and pelvis as well. With this updated PDIP-SOS CMR technique, it is now possible to simultaneously and accurately assess vascular calcifications in both the aorto-iliac and ilio-femoral regions with a single, $<6$-min oblique coronal acquisition.

Across all patients at both magnetic field strengths, CMR slightly underestimated calcification volumes (linear regression slopes of $\sim 0.89$ and $\sim 0.93$ for ilio-femoral and aorto-iliac regions) compared with CTA. This discrepancy might be explained by the fact that blooming artifact artifactually enlarges dense calcifications on CT images [20]. Alternatively, it is conceivable that the surface regions of a calcification contain mobile water spins that produce detectable signal intensity, which would decrease their apparent volume with PDIP-SOS CMR.

A limitation of our study was that contrast-enhanced CTA was used as a reference standard, whereas non-contrast CT would be preferred. However, at the two institutions used for this study, non-contrast CT series were acquired at 3 to $5-\mathrm{mm}$ slice thickness, too thick for volumetric evaluation, compared with 0.6 to 1-mm slice thickness for CTA. Also, PDIP-SOS CMR scan times were substantially longer than is the case with CT. Scan time can be greatly reduced (e.g. to $30 \mathrm{~s}$ or less) by acquiring thicker slices and using fewer radial views at the expense of more partial volume averaging, decreased SNR and increased radial streak artifacts. While this accelerated approach would reduce sensitivity for very small calcifications, it is unlikely that detection of bulky, clinically significant calcifications would be adversely affected. Finally, our study demonstrated that imaging of both aorto-iliac and ilio-femoral calcifications is accurate at 3 Tesla. While imaging of ilio-femoral calcifications is also accurate at 1.5 Tesla, the study was underpowered $(n=1)$ at this field strength to draw any conclusions about aorto-iliac calcifications.

\section{Conclusion}

Using PDIP-SOS CMR, aorto-iliac and ilio-femoral calcifications could be simultaneously evaluated at 3 Tesla in less than six minutes with excellent correlation and agreement to CTA. Lesion size was not substantially affected by magnetic field strength. Our results suggest that PDIP-SOS CMR provides a reliable alternative to CT for pre-interventional evaluation of peripheral vascular calcium burden.

\section{Abbreviations \\ CMR: cardiovascular magnetic resonance; CT: computed tomography; CTA: computed tomographic angiography; PAD: peripheral arterial disease; PDIP-SOS: proton density-weighted, in-phase stack-of-stars; QISS: Quiescent- Interval Slice-Selective; SNR: signal-to-noise ratio}

\section{Acknowledgements}

We would like to thank Dr. Wei Li and Maria Carr for assisting with data collection and analysis.

\section{Funding}

Research support, NIH grants R01 HL130093 and R21 HL126015. Research support, Siemens Healthineers. Research support, Department of Radiology, NorthShore University HealthSystem.

\section{Authors' contributions}

RE: participated in all aspects of the study and is the guarantor of study integrity. AS: assisted with image analysis and manuscript review. PA: assisted with image analysis and manuscript review. JC: assisted with patient recruitment and manuscript review. OM: assisted with patient recruitment and manuscript review. SG: assisted with pulse sequence implementation and manuscript review. IK: assisted with pulse sequence implementation, statistical analysis and manuscript review. All authors read and approved the manuscript.

\section{Ethical approval and consent to participate}

The prospective volunteer/patient study was approved by the NorthShore University HealthSystem and Northwestern Memorial Hospital Institutional Review Boards (IRB).

\section{Consent for publication}

Consent for publication of their individual details and accompanying images in this manuscript was prospectively provided in all 43 subjects. No protected health information for any subject is given in this manuscript. The tables and figures are all original and have never been submitted or published elsewhere.

\section{Competing interests}

RE: Research support and invention licensing agreement, Siemens Healthcare. SG: Employee, Siemens Healthineers. There were no potential financial conflicts for the other authors. There were no non-financial. conflicts of interest for any of the authors.

\section{Publisher's Note}

Springer Nature remains neutral with regard to jurisdictional claims in published maps and institutional affiliations.

\section{Author details}

${ }^{1}$ Radiology, Northwestern Memorial Hospital, Chicago, IL, USA. ${ }^{2}$ Radiology, Northshore University HealthSystem, Walgreen Building, G534, 2650 Ridge Avenue, Evanston, IL 60201, USA. ${ }^{3}$ Radiology, University of Chicago Pritzker School of Medicine, Chicago, IL, USA. ${ }^{4}$ Siemens Healthineers, Chicago, IL, USA. ${ }^{5}$ Surgery, Northshore University HealthSystem, Evanston, IL, USA. 
Received: 6 November 2017 Accepted: 19 July 2018

Published online: 06 August 2018

\section{References}

1. Met R, Bipat S, Legemate DA, Reekers JA, Koelemay MJ. Diagnostic performance of computed tomography angiography in peripheral arterial disease: a systematic review and meta-analysis. JAMA. 2009;301:415-24.

2. Menke J, Larsen J. Meta-analysis: accuracy of contrast-enhanced magnetic resonance angiography for assessing steno-occlusions in peripheral arterial disease. Ann Intern Med. 2010:153:325-34

3. Tranche-Iparraguirre S, Marín-Iranzo R, Fernández-de Sanmamed R, Riesgo-García A, Hevia-Rodríguez E, García-Casas JB. Peripheral arterial disease and kidney failure: a frequent association. Nefrologia. 2012;32:313-20. https://doi.org/10.3265/Nefrologia.pre2011.Nov.11172. Epub 2012 Jan 27

4. Miyazaki M, Lee VS. Nonenhanced MR angiography. Radiology. 2008;248:20-43. https://doi.org/10.1148/radiol.2481071497.

5. Singh DK, Winocour P, Summerhayes B, Kaniyur S, Viljoen A, Sivakumar G, Farrington K. Prevalence and progression of peripheral vascular calcification in type 2 diabetes subjects with preserved kidney function. Diabetes Res Clin Pract. 2012;97:158-65. https://doi.org/10.1016/j.diabres.2012.01.038. Epub 2012 Mar 3

6. Ouwendijk R, Kock MC, van Dijk LC, van Sambeek MR, Stijnen T, Hunink MG. Vessel wall calcifications at multi-detector row $\mathrm{CT}$ angiography in patients with peripheral arterial disease: effect on clinical utility and clinical predictors. Radiology. 2006;241:603-8.

7. Niskanen L, Siitonen O, Suhonen M, Uusitupa MI. Medial artery calcification predicts cardiovascular mortality in patients with NIDDM. Diabetes Care. 1994;17:1252-6.

8. Guzman RJ, Brinkley DM, Schumacher PM, Donahue RM, Beavers H, Qin X. Tibial artery calcification as a marker of amputation risk in patients with peripheral arterial disease. J Am Coll Cardiol. 2008;51:1967-74.

9. Pentecost MJ, Criqui MH, Dorros G, et al. Guidelines for peripheral percutaneous transluminal angioplasty of the abdominal aorta and lower extremity vessels. A statement for health professionals from a special writing group of the councils on cardiovascular radiology, arteriosclerosis, cardio-thoracic and vascular surgery, clinical cardiology, and epidemiology and prevention, the American Heart Association. Circulation. 1994;89:511-31.

10. Manunga JM, Gloviczki P, Oderich GS, Kalra M, Duncan AA, Fleming MD, Bower TC. Femoral artery calcification as a determinant of success for percutaneous access for endovascular abdominal aortic aneurysm repair. J Vasc Surg. 2013 Jul 2

11. McDermott MM, Liu K, Carroll TJ, et al. Superficial femoral artery plaque and functional performance in peripheral arterial disease: walking and leg circulation study (WALCS III). JACC Cardiovasc Imaging. 2011;4:730-9. https://doi.org/10.1016/j.jcmg.2011.04.009.

12. Tenner MS, Spiller M, Koenig SH, Valsamis MP, Childress S, Brown RD 3rd, Kasoff SS. Calcification can shorten T2, but not T1, at magnetic resonance imaging fields. Investig Radiol. 1995;30(6):345-53.

13. Edelman RR, Flanagan O, Giri S, Koktzoglou I. Neutral contrast MRI for the detection of peripheral arterial wall calcifications. J Cardiovasc Magn Reson. 2014;16(Suppl 1):O76. https://doi.org/10.1186/1532-429X-16-S1-076.

14. Yang Q, Liu J, Barnes SRS, et al. Imaging the vessel wall in major peripheral arteries using susceptibility weighted imaging. J Magn Reson Imaging. 2009:30(2):357-65.

15. Edelman RR, Sheehan JJ, Dunkle E, Schindler N, Carr J, Koktzoglou I. Quiescent-interval single-shot unenhanced magnetic resonance angiography of peripheral vascular disease: technical considerations and clinical feasibility. Magn Reson Med. 2010;63:951-8.

16. Ferreira Botelho MP, Koktzoglou I, Collins JD, Giri S, Carr JC, Gupta N, Edelman RR. MR imaging of iliofemoral peripheral vascular calcifications using proton density-weighted, in-phase three-dimensional stack-of-stars gradient echo. Magn Reson Med 2017 Jun;77(6):2146-2152. https://doi.org/ 10.1002/mrm.26295. Epub 2016 Jun 14

17. Cicchetti DV. Guidelines, criteria, and rules of thumb for evaluating normed and standardized assessment instruments in psychology. Psychological Assessment. 1994;6:284-90. https://doi.org/10.1037/1040-3590.6.4.284.
18. Glover GH, Pauly JM. Projection reconstruction techniques for reduction of motion effects in MRI. Magn Reson Med. 1992;28:275-89.

19. Block KT, Chandarana H, Milla S, et al. Towards routine clinical use of radial stack-of-stars 3D gradient-echo sequences for reducing motion sensitivity. Journal of the Korean Soc Magnet Resona in Med. 2014;18(2):87-106.

20. Fleischmann D, Hallett RL, Rubin GD. CT angiography of peripheral arterial disease. J Vasc Interv Radiol. 2006 Jan;17(1):3-26.

\section{Ready to submit your research? Choose BMC and benefit from:}

- fast, convenient online submission

- thorough peer review by experienced researchers in your field

- rapid publication on acceptance

- support for research data, including large and complex data types

- gold Open Access which fosters wider collaboration and increased citations

- maximum visibility for your research: over $100 \mathrm{M}$ website views per year

At BMC, research is always in progress.

Learn more biomedcentral.com/submissions 